\title{
Metformin as a Potential Adjuvant Antimicrobial Agent Against Multidrug Resistant Bacteria
}

\author{
Majed M Masadeh' \\ Karem H Alzoubi $\mathbb{D}^{2}$ \\ Majd M Masadeh' \\ Zainah $O$ Aburashed (iD) ${ }^{2}$ \\ 'Department of Pharmaceutical \\ Technology, Faculty of Pharmacy, Jordan \\ University of Science and Technology, \\ Irbid, 22I I0, Jordan; ${ }^{2}$ Department of \\ Clinical Pharmacy, Faculty of Pharmacy, \\ Jordan University of Science and \\ Technology, Irbid, Jordan
}

\begin{abstract}
Introduction: The continuous increase in the incidence of bacterial resistance to existing antibiotics represents a worldwide health burden. A surrogate strategy to combat such crisis is to find compounds that restore the antimicrobial activity of the already existing antibiotics against multidrug resistant bacteria. Metformin is a commonly used antidiabetic medication. It has proven benefits in other diseases including cancer, aging-related and infectious diseases. In this study, the potential effect of metformin as an adjuvant therapy to antibiotics was investigated.
\end{abstract}

Methods: Two multidrug resistant bacterial strains were used; methicillin-resistant Staphylococcus aureus (MRSA; ATCC 33,591) and multidrug resistant Pseudomonas aeruginosa (ATCC BAA-2114). To assess its efficacy, metformin was combined with several antibiotics: levofloxacin, chloramphenicol, rifampicin, ampicillin, and doxycycline. The antibacterial effect of metformin was tested using the micro broth dilution method. The minimum inhibitory concentration (MIC) was also measured. Cytotoxicity studies were also performed on mammalian cells to assess its safety.

Results: Metformin exhibited an antibacterial effect when combined with the antibiotics on the two tested strains. It also showed low toxicity on the mammalian cells. Moreover, synergetic studies showed that metformin enhanced the effect of the combined antibiotics, as these combinations provide either a synergistic or additive effect with significant reduction in the MIC.

Conclusion: Metformin exerts an adjuvant antibacterial effect; thus, it could be a possible candidate as an adjuvant therapy to reduce antimicrobial resistance.

Keywords: metformin, antibacterial resistance, multidrug resistant bacteria, non-antibiotics, MIC, adjuvant

\section{Introduction}

For the past two decades, huge efforts were made to combat infections caused by resistant bacteria. ${ }^{1}$ Despite the implementation of rigorous strategies to reduce their spread, the fast, widespread dissemination of infections caused by highly resistant bacteria is still a major global health concern. ${ }^{2}$ Paralleling the development of resistance in Gram-positive bacteria, resistance in Gram-negative strains are also a growing problem. ${ }^{3}$ Around 700,000 people die yearly due to infections caused by multidrug resistant (MDR) Gram-positive and Gram-negative bacteria, and this number is expected to further increase and reach up to 10 million deaths by $2050 .{ }^{4,5}$ The negative impact of infections caused by MDR is extended to impact not only in the health field, but also the economic field due to longer hospitalization and greater costs of longer care when compared with infections caused by
Correspondence: Majed M Masadeh Department of Pharmaceutical Technology, Jordan University of Science and Technology, Irbid, 22110, Jordan Email mmmasadeh@just.edu.jo 
antimicrobial susceptible bacteria. ${ }^{6}$ The number of antimicrobial medications discovered within the last two decades is much lower compared with previous discoveries. ${ }^{7}$ This might be due to the high cost required to develop new antimicrobials, justifying why many pharmaceutical companies withdraw from researching new antimicrobial agents. ${ }^{8,9}$ Among the strategies used to reduce resistance, is combination among the antibiotics. However, this strategy is failing with the increasing cost and adverse effects of such combinations. ${ }^{1,10}$ Furthermore, irrational use of antibiotics combinations may worsen the current scenario of bacterial resistance. ${ }^{11}$ On the other hand, the idea of reversing bacterial resistance by means of non-antibiotic antimicrobials is now taking place and seems to be favorable. ${ }^{10,12}$ A variety of medicinal compounds that were used originally for the management of noninfectious pathologic conditions exhibit an antimicrobial activity against different microorganisms. ${ }^{1,13}$ These medications, which are pharmacologically not considered to be conventional antibiotics but exert antimicrobial activity, are called non-antibiotics. ${ }^{14,15}$ Non-antibiotic antimicrobial agents were shown to obviate, lessen and reverse bacterial resistance to the classical antibiotics. ${ }^{16}$ Moreover, non-antibiotic medications were shown to enhance the in vitro activity of the classical antibiotics, making bacteria more susceptible to the formerly conventional ineffective therapy. ${ }^{17-20}$ Medications including antihistamine, neuroleptics, non-steroidal anti-inflammatory medications and the antidiabetic drug metformin were found to have a degree of antimicrobial activity. ${ }^{21-24}$

Metformin (dimethylbiguanide) is a commonly used antihyperglycemic medication. ${ }^{24}$ Its tolerably manageable side-effect profile along with its non-antidiabetic benefits, including the reduction of cardiovascular events, make metformin the first-line therapy for most patients with type two diabetes. ${ }^{14}$ In several studies, diabetic patients receiving metformin were less likely to have infections, ${ }^{25,26}$ suggesting a possible antimicrobial benefit of metformin. ${ }^{14,27}$ In a recent study, metformin exhibited a potential synergistic effect when combined with tetracycline group against a highly resistant strain of $E$. coli in an animal model. ${ }^{28}$

Although not fully understood, metformin can exert adjuvant antimicrobial activity through several possible mechanisms, including potentiation of the activity of the antibiotics, modifying the immune response of the host cells to the infection, and its ability to increase the intracellular accumulation of different antibiotics through disrupting the outer membranes of bacteria. ${ }^{12,28,29}$ Thus, metformin can possibly overcome bacterial resistance through not only enhancing the antimicrobial effect of the antibiotics, but also through its immunomodulatory effect. $^{28}$

In the current study, we investigated the possible antimicrobial activity of metformin in vitro. Two highly resistant bacterial strains were used: the Gram-positive strain methicillin resistant Staphylococcus aureus (MRSA), and to test its effect on Gram-negative bacteria, multidrug resistant (MDR) Pseudomonas aeruginosa were used. Micro broth dilution method was used to assess the antibacterial effect of metformin. This study could be of clinical significance as metformin could prove to be a potential antimicrobial agent to be used for antibioticresistant bacteria.

\section{Materials and Methods \\ Chemicals and Materials}

All chemical reagents, metformin and the antibiotics were obtained from Sigma Chemical Co. (St. Louis, MO, USA). Manufacturer's recommendations were followed for better handling of these reagents and materials.

\section{Bacterial Strains}

Two bacterial strains were used for testing and determining the antimicrobial activity of metformin: methicillin resistant Staphylococcus aureus (MRSA) ATCC 33,591 and the multidrug resistant (MDR) Pseudomonas aeruginosa (P. aeruginosa) ATCC BAA-2114. Bacterial strains were derived from American Type Tissue Culture Collection (ATCC; Manassas, VA, USA).

\section{Bacterial Susceptibility Assay}

The micro broth dilution method, using 96-well plates, described in Clinical and Laboratory Standards Institute (CLSI) was adopted to test bacterial susceptibility., ${ }^{2,30}$ Bacterial susceptibility was assessed through measuring the minimum inhibitory concentration (MIC) for metformin and for the tested antibacterial agents. MIC can be defined as the lowest concentration of the antibiotic required to visibly inhibit bacterial growth after overnight incubation. ${ }^{31}$ In brief, bacteria from the two tested strains were removed from their frozen stocks and grown overnightin Mueller Hinton Broth $(\mathrm{MH})$ and diluted to $10^{6}$ $\mathrm{CFU} / \mathrm{mL}$ in a culture media before being used. After that, $50 \mu \mathrm{L}$ of 2 -fold dilution of metformin (in 
a concentration of $500 \mu \mathrm{M}$ ) were added to $50 \mu \mathrm{L}$ of each bacterial suspension in its mid-log phase and placed on 96well plates, which were then incubated at $37^{\circ} \mathrm{C}$ for $18 \mathrm{~h}$ to read the MIC. The positive control contained $50 \mu \mathrm{L}$ of the agar media and $50 \mu \mathrm{L}$ of the bacterial suspension. The negative control consisted of $100 \mu \mathrm{L}$ of sterilized agar. MIC was measured at a wavelength of $600 \mathrm{~nm}$ using an enzyme linked immunosorbent assay (ELISA) microplate reader (Epoch TM; BioTek, Winooski, VT, USA). The minimum bactericidal concentration (MBC) was measured after that. Bacterial aliquots (10 $\mu \mathrm{L}$ of the well content) were obtained from the wells that showed no visible turbidity, spread on Mueller Hinton Broth agar media (MHB; Oxoid Ltd, Basingstoke, UK), and allowed for 24 or 48 $\mathrm{h}$ incubation at $37^{\circ} \mathrm{C}$. The minimum concentration that leads to less than $1 \%$ survival of the bacterial subculture was considered as the MBC. All MIC and MBC calculations were made in triplicate.

\section{Synergistic Checkerboard Assay}

The synergistic effect of combining metformin with other antibiotics was tested using two bacterial strains: MRSA ATCC 33,591 and P. aeruginosa ATCC-2114. Five antimicrobial agents were used: rifampicin, levofloxacin, chloramphenicol, ampicillin, and doxycycline. The procedure was performed as mentioned in previous studies. ${ }^{5,32}$ Using 96-well microplates, $25 \mu \mathrm{L}$ of metformin and $25 \mu \mathrm{L}$ of one of the mentioned antibiotics were added to $50 \mu \mathrm{L}$ of $5 \times 10^{5} \mathrm{CFU} / \mathrm{mL}$ of each bacterial strain suspension on each well. The microplates were then incubated for $24 \mathrm{~h}$ at $37^{\circ}$ C. The MICs were then measured at $600 \mathrm{~nm}$ using an ELISA microplate reader (EpochTM, BioTeck, Winooski, VT, USA). All the procedures were conducted in triplicate.

Finally, the fractional inhibitory concentration (FIC) was calculated for each combination by adding the FIC of metformin to the FIC of the antibiotic. Determining FICs value were made by dividing the MIC of each drug in combination by the MIC of the same drug alone. The reaction was classified as synergetic if the FIC $\leq 0.5$, additive if greater than 0.5 or lower/equal than 1 , indifferent if greater than 1 but less than 2 and antagonistic if greater or equal to $2 .^{32}$

\section{Mammalian Cell Cytotoxicity}

To test the cytotoxicity of metformin and the other tested antimicrobial agents, VERO cell culture, a commonly used mammalian cell culture obtained from the kidneys of the African monkey were used. ${ }^{33}$ VERO cell cultures (Sigma-
Aldrich, St. Louis, MO, USA) were first allowed to grow in RPMI 1640 media. This mammalian growth media contains $1 \% \mathrm{v} / \mathrm{v}$ concentration of streptomycin and penicillin and $10 \%$ of fetal bovine serum. Cells were cultured as single layers (monolayers) in $95 \%$ air, $5 \% \mathrm{CO}_{2}$ at $37^{\circ} \mathrm{C}$. VERO cells were then transferred to the 96-well microtiter plate to give a density of $5 \times 10^{3}$ cells/well and incubated for $18 \mathrm{~h}$ with metformin and the tested antibiotics in a fixed dose combination equivalent to the MIC or multiple of the MIC of metformin and the antibiotics tested as shown in Table 1 . Thereafter, $20 \mu \mathrm{L}$ with a final concentration of $5 \mathrm{mg} / \mathrm{mL}$ from 3-(4,5-dimethylthiazol-2-yl)2,5-diphenyltetrazolium bromide (MTT) was added to each well and incubated for an additional $6 \mathrm{~h}$. The viability of the mammalian cells was tested by changing the color of MTT into a purple color, suggesting the production of formazan crystals. Following extraction, formazan crystals were dissolved in $100 \mu \mathrm{L}$ of dimethyl sulfoxide (DMSO). The absorbance of the extracted and dissolved formazan crystals was checked at a wavelength of $550 \mathrm{~nm}$ using an ELISA reader (EpochTM, BioTeck, Winooski, VT, USA).

\section{Results}

\section{Bacterial Susceptibility Against Metformin and the Used Antimicrobials}

The microbroth-dilution method was used to study the possible adjuvant antibacterial effect of metformin. Two highly resistant bacterial species were used; one Gram-positive as well as one Gram-negative bacteria (MRSA and $P$. aeruginosa, respectively). Metformin exerted a potent antimicrobial activity on the two tested strains when combined with the other antibacterial agents. The MIC for metformin alone was the same $(500 \mu \mathrm{M})$ for all the tested strains. For the other studied antibacterial agents, MRSA showed the highest sensitivity and the lowest MIC with rifampicin, and the lowest sensitivity and the highest MIC was against chloramphenicol. On the other hand, the highly resistant Gram-negative bacteria, $P$. aeruginosa, had the highest sensitivity against levofloxacin and exerted the lowest sensitivity against ampicillin, manifested by high MIC required to inhibit bacterial growth. The MBC readings of metformin measured against the studied strains were equal to the MIC for all the strains, suggesting a bactericidal potential of metformin (Table 1).

\section{Synergistic Checkerboard Assay}

Given the possible antibacterial effect of metformin, we examined the probable synergistic effect when metformin 


\begin{tabular}{|c|c|c|}
\hline 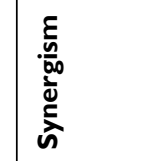 & 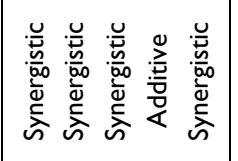 & 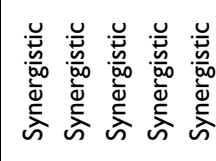 \\
\hline$\overline{\underline{U}} \hat{\Sigma}_{\underline{E}}$ & $\frac{n}{0} \frac{}{0} \frac{}{0} \frac{\mathfrak{0}}{0} \frac{\mathrm{N}}{0}$ & 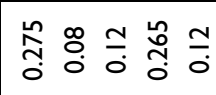 \\
\hline 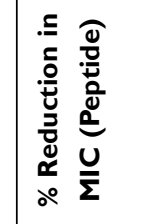 & 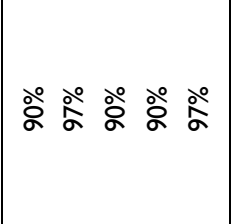 & 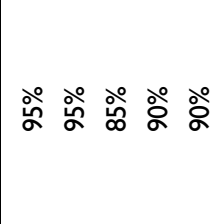 \\
\hline 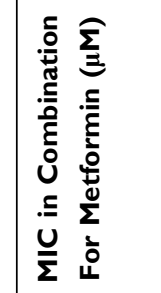 & 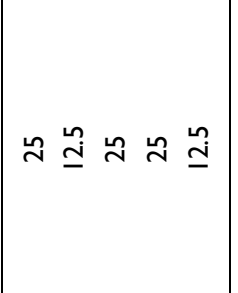 & 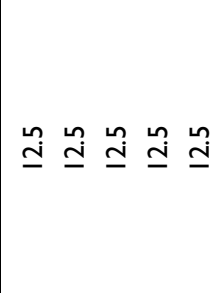 \\
\hline 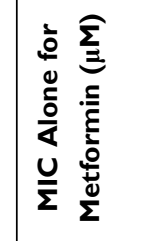 & 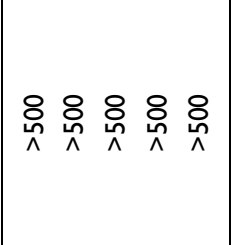 & 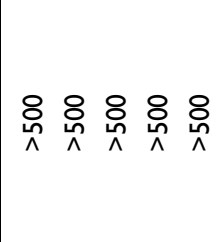 \\
\hline 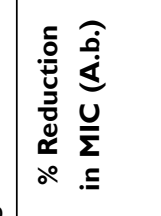 & 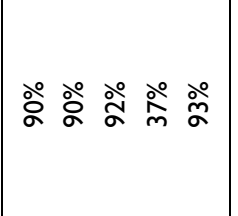 & 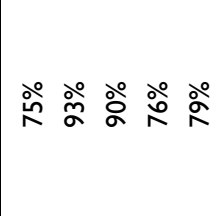 \\
\hline 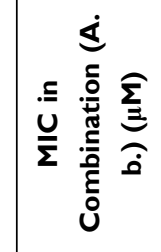 & 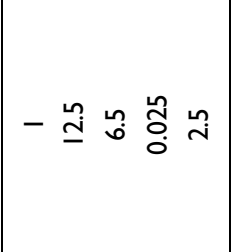 & $m \stackrel{n}{\underline{i}}$ in $\simeq \stackrel{\text { S }}{\mathrm{i}}$ \\
\hline 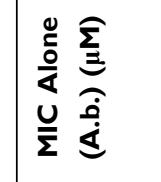 & 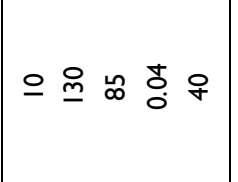 & 융ㅇㅅㅅㅇㅇㅇ \\
\hline 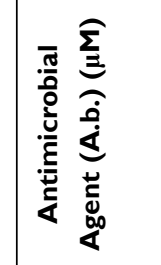 & 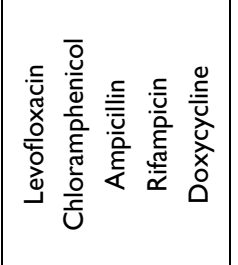 & 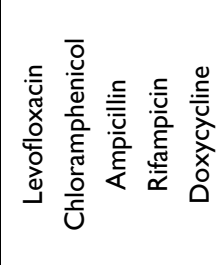 \\
\hline 焉 & 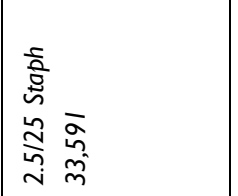 & 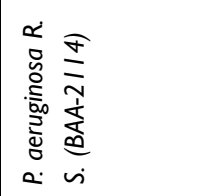 \\
\hline
\end{tabular}

was combined with the other antibacterial agents using checkerboard method. Five antimicrobial agents were used for the synergistic studies: levofloxacin, rifampicin chloramphenicol, ampicillin, and doxycycline. The resistant bacterial strains used were MRSA ATCC 33,591 and MDR P. aeruginosa ATCC BAA-2114. First, FIC indices were calculated, and values were analyzed and inferenced. The combinations were interpreted, based on EUCAST as synergistic (FIC $\leq 0.5$ ), additive $0.5<$ FIC index $\leq 1$, indifferent $1<$ FIC $<2$, or antagonistic (FIC $\geq 2$ ). ${ }^{31-34}$ As illustrated in Table 1, the combinations of metformin with the tested antimicrobial agents, against MRSA, were all synergistic, with the exception of rifampicin which was additive. Combining metformin with doxycycline resulted in the highest reduction in the antibiotic MIC with approximately $93 \%$ reduction in doxycycline MIC. On the other hand, rifampicin-metformin combination had an additive effect with the lowest reduction in the MIC of the tested agents, which was around $37 \%$ of rifampicin MIC. In addition, as shown from the same table, metformin showed a synergistic effect against $P$. aeruginosa ATCC BAA2114 when combined with all tested antibiotics. For the same tested strain, the highest antibiotic MIC reduction was $93 \%$ (chloramphenicol-metformin) accompanied with a $97 \%$ drop in metformin MIC. On the other hand, combining metformin with levofloxacin resulted in $75 \%$ reduction of levofloxacin MIC, which was the lowest reduction in the MIC of the studied medications. At the same time, adding metformin to all tested agents, for the two studied species, was associated with a decline in the MIC of metformin. The highest reduction of metformin MIC was when combined with chloramphenicol and doxycycline against MRSA (97\% reduction in metformin MIC) and the lowest reduction was approximately $85 \%$ when added to ampicillin against MDR $P$. aeruginosa. Overall, the combination of metformin with the antibacterial agents had either synergetic or additive effects or the MIC concentration for all studied antimicrobials was reduced when combined with metformin. This drop in antibiotics-MIC was also accompanied with a decline in metformin MIC.

\section{Mammalian Cell Cytotoxicity Assay}

Using the MTT assay method, the safety of metformin was investigated on the mammalian cells using VERO cell lines. The cytotoxicity of metformin on eukaryotic cells has been investigated when used in combination with various antibiotics. As illustrated in Figure 1, to induce $50 \%$ cytolysis in the mammalian cells, more than three 

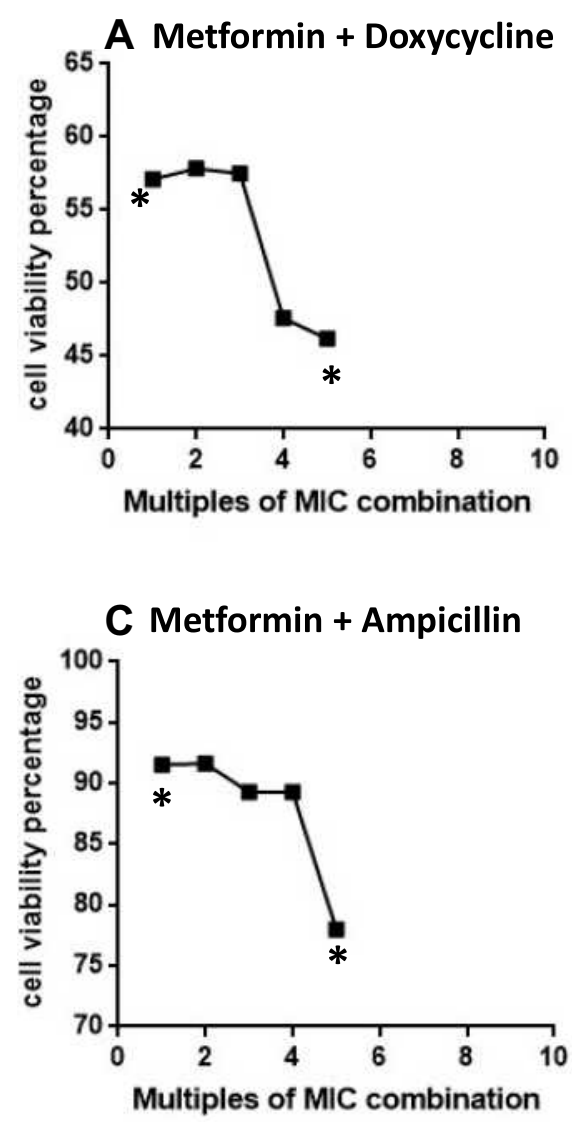
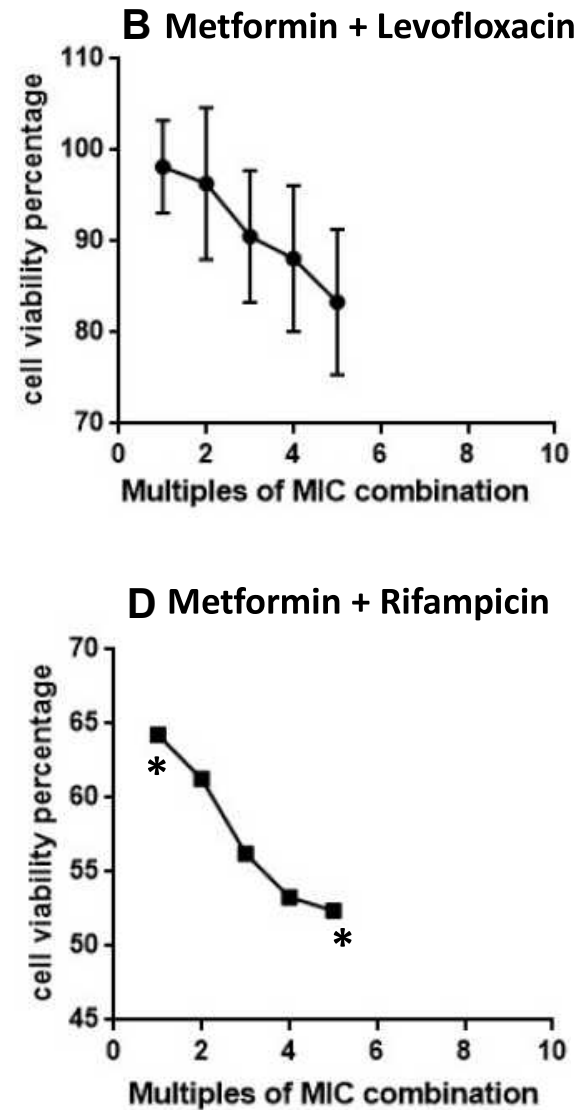

Figure I The cytotoxicity of combinations of metformin and tested antibiotics in VERO cell line expressed as cell viability percentage, A. Metformin + Doxycycline, B. Metformin + Levofloxacin, C. Metformin + Ampicillin, and D. Metformin + Rifampicin. Dose of combination is expressed as multiples of MIC of the combination of metformin and the tested antibiotic. Points between the two stars $\left(^{*}\right)$ are significantly different from baseline (zero dose) cell viability using unpaired $t$-test and P<0.05. Values are expressed as Mean \pm SD.

times the MIC of the combination metformin-doxycycline was required. For the measured MIC of this combination, approximately $57 \%$ of the cells remained viable. This suggests high safety of this combination. It is worth noting that the addition of metformin to doxycycline resulted in a remarkable reduction in the MIC for both metformin and doxycycline. This further enhanced the safety of metformin when used as an adjuvant with this antibacterial agent since lower doses of doxycycline can be given (Figure 1A). Other combinations were also tested. When adding metformin to the fluoroquinolone "levofloxacin" and to ampicillin, cells were viable even when using high concentration of the combinations, as more than $85 \%$ of the cells remained alive (with more than four times of the MIC for the two combinations) (Figure $1 \mathrm{~B}$ and $\mathrm{C}$ ).

Adding metformin to the remaining antibiotics rifampicin and chloramphenicol does not produce a significant cytotoxicity for the mammalian cells either. When applying high concentrations of these two combinations on VERO cell culture ( 4 times the MIC), more than 50\% and $75 \%$ of the cells survived with rifampicin and chloramphenicol, respectively (Figure 1D and E). As a result, these combinations are safe and do not produce a significant harm to the mammalian cells when used in standard concentrations.

\section{Discussion}

In recent years, the emergence and fast spread of antibiotic resistance has become a severe threat to health all over the globe. ${ }^{35,36}$ Classical methods that were aimed to contain or slow the rapid progression of bacterial resistance through better antibiotic prescribing policies, have become insufficient at the global level. ${ }^{37,38}$ Urgent measures along with identification of novel adjuvant strategies to restore the efficacy of the preexisting antibiotics and ensure better clinical outcomes, in a cost-effective manner, are highly needed. ${ }^{39}$ Of the strategies that were extensively studied to reduce bacterial resistance is the use of non-antibiotic medications that possess an antimicrobial effect. $^{16,20,40,41}$ Metformin, a mainstay in the treatment of 
type two diabetes, was found, in addition to being a glucose-lowering medication, to possess an adjuvant antimicrobial effect either alone or when combined with other antibiotics. $^{42}$ It is generally a safe and a welltolerated medication with gastrointestinal symptoms being the most reported adverse effects. ${ }^{43}$ It is one of the few approved medications for type two diabetes in children. ${ }^{44}$ The usual therapeutic dose of metformin ranges from 500-2500 $\mathrm{mg}$ daily. ${ }^{45}$ Several advance studies, both in vivo and in vitro, showed that metformin has an antimicrobial effect against a variety of microorganisms including mycobacterium tuberculosis, Grampositive and Gram-negative bacteria and that metformin can be a possible adjunct therapy with other antimicrobials. $^{28,34,46}$ The major finding of the current project is that metformin showed a possible antimicrobial effect when studied in combination with other antibiotics, and that metformin can be used to reduce bacterial resistance to already existing antimicrobials.

In our study, two highly resistant strains of Grampositive and Gram-negative bacteria were studied, MRSA and multidrug $P$. aeruginosa, respectively. Metformin was able to reduce the resistance of these two strains to the tested antibiotics namely, doxycycline, levofloxacin, ampicillin, chloramphenicol, and rifampicin. Measuring MIC was used as a tool to demonstrate bacterial resistance. Furthermore, these combinations (metformin-tested antibiotics) provided either a synergistic or an additive effect; suggesting that metformin efficiently enhances and potentiates the activity of these antibiotics.

Consistent with our findings, several previous published studies showed that metformin has the potential to be used in combination with other antibiotics, ${ }^{14,27-29}$ and metformin can reduce the resistance of bacteria and efficiently restore the efficacy of the antibiotics. ${ }^{28}$ These studies along with other studies confirm the antimicrobial potential of metformin either alone or in combination, not only with antibacterial, ${ }^{24,28,47}$ but also with antifungal, ${ }^{48}$ antiprotozoal, and anti-tuberculosis effects. $^{49,50}$

Another important aspect is the safety of metformin when combined with other antibiotics. Metformin is generally a safe medication with few, manageable side effects. $^{28}$ Because of its low side-effects profile, in addition to its pleiotropic effects including weight loss and cardiovascular risk reduction, it is by far the preferred first-line therapy for most type two diabetic patients. ${ }^{51-54}$ In the current study, metformin safety was evaluated using the VERO mammalian cell line. VERO cell viability was measured as a tool to assess the safety of metformin. The viability of the cells remained satisfactory and high despite using high concentrations of metformin, suggesting a high safety profile of metformin. When combined with rifampicin and doxycycline, the lowest viability was detected, suggesting that the lowest safety was with these two combinations. This can be explained by the harm induced by these two antibiotics and not related to metformin. Our findings are in accordance with other formerly published studies that showed a favorable safety profile for metformin. ${ }^{54-57}$ Although there are other medications, including non-steroidal anti-inflammatory medications (NSAIDs), antidepressants and some neuroleptics that exert a potential antimicrobial effect, ${ }^{16,58}$ metformin was the selected medication for this study. It was chosen not only because of its superior efficacy as an adjuvant antibacterial, but also because of its preferred safety profile. $^{59}$

Although the exact mechanism underlying the antibacterial effect of metformin is not fully understood, ${ }^{28}$ several mechanisms have been proposed; one of these mechanisms is the ability of metformin to enhance the antimicrobial effect of the antibiotics. ${ }^{60}$ Furthermore, metformin disrupts the outer membrane of the bacteria which affects the permeability of the antibacterial agents, allowing better penetration of antibiotics. ${ }^{28}$ Moreover, metformin reduces the inflammatory effect induced by bacteria. $^{61}$ Taking all these possible mechanisms together, metformin enhances the accumulation of the antibiotics in the bacteria, thus reducing microbial resistance. Although we have not investigated the mechanisms of the antimicrobial effect of metformin, our future studies will be directed toward better understanding the mechanisms which underlie this effect.

In summary, metformin, in combination with other antibiotics, exerts an antimicrobial effect when studied on different bacterial strains. It may be promising to consider metformin as an adjuvant therapy to tackle bacteria. Several studies are still needed for better understanding the mechanisms underlying this preventive effect of metformin, but the preliminary findings from our study highly support the use of metformin as an adjuvant to antibiotics to reduce bacterial resistance.

\section{Acknowledgment}

This research was funded by the Deanship of Research at Jordan University of Science and Technology. 


\section{Disclosure}

The authors declare no conflict of interest.

\section{References}

1. Amaral L, Viveiros M, Fau - Kristiansen JE, Kristiansen JE. "NonAntibiotics": alternative therapy for the management of MDRTB and MRSA in economically disadvantaged countries. Curr Drug Targets. 2006;7(7):887-891. doi:10.2174/138945006777709539

2. Ouhara K, Komatsuzawa H, Kawai T, et al. Increased resistance to cationic antimicrobial peptide LL-37 in methicillin-resistant strains of Staphylococcus aureus. J Antimicrob Chemother. 2008;61 (6):1266-1269. doi:10.1093/jac/dkn106

3. Thomson JM, Bonomo RA. The threat of antibiotic resistance in gram-negative pathogenic bacteria: beta-lactams in peril! Curr Opin Microbiol. 2005;8(5):518-524. doi:10.1016/j.mib.2005.08.014

4. Organization WH. 2019.

5. Al Tall Y, Abualhaijaa A, Qaoud MT, Alsaggar M, Masadeh M, Alzoubi KH. The ultrashort peptide OW: a new antibiotic adjuvant. Curr Pharm Biotechnol. 2019;20(9):745-754. doi:10.2174/ 1389201020666190618111252

6. Lee NY, Lee HC, Fau - Ko N-Y, et al. Clinical and economic impact of multidrug resistance in nosocomial Acinetobacter baumannii bacteremia. Infect Control Hosp Epidemiol. 2007;28(6):713-719.

7. Renwick M, Mossialos E. What are the economic barriers of antibiotic R\&D and how can we overcome them? Expert Opin Drug Discov. 2018;13(10):889-892.

8. Silver LL. Challenges of antibacterial discovery. Clin Microbiol Rev. 2011;24(1):71-109. doi:10.1128/CMR.00030-10

9. Coates ARM, Hu Y, Holt J, Yeh P. Antibiotic combination therapy against resistant bacterial infections: synergy, rejuvenation and resistance reduction. Expert Rev Anti Infect Ther. 2020;18(1):5-15.

10. Kristiansen JE, Hendricks O, Delvin T, et al. Reversal of resistance in microorganisms by help of non-antibiotics. J Antimicrob Chemother. 2007;59(6):1271-1279. doi:10.1093/jac/dkm071

11. Ahmed A, Azim A, Gurjar M, Baronia AK. Current concepts in combination antibiotic therapy for critically ill patients. Indian $J$ Crit Care Med. 2014;18(5):310-314. doi:10.4103/09725229.132495

12. Meherunisa JS, Seth V. Study of metformin effect on antimicrobial property. Int Archi BioMed Clin Res. 2018;4:85-87.

13. Cederlund H, Mårdh PA. Antibacterial activities of non-antibiotic drugs. J Antimicrob Chemother. 1993;32(3):355-365.

14. Tr P, Patil S, Patil S, Patil A. Antimicrobial potential of metformin. Int J Pharmacogn Phytochem Res. 2019;11(3):230-234.

15. Karak P, Kumar KA, Mazumdar K, Mookerjee M, Dastidar S. Antibacterial potential of an antispasmodic drug dicyclomine hydrochloride. Indian J Med Res. 2003;118:192-196.

16. Lagadinou M, Onisor MO, Rigas A, et al. Antimicrobial properties on non-antibiotic drugs in the era of increased bacterial resistance. Antibiotics. 2020;9(3):107. doi:10.3390/antibiotics9030107

17. Kristiansen JE, Amaral L. The potential management of resistant infections with non-antibiotics. J Antimicrob Chemother. 1997;40 (3):319-327.

18. Amaral L, Kristiansen JE, Fau - Frolund Thomsen V, Frolund Thomsen V, Fau - Markovich B, Markovich B. The effects of chlorpromazine on the outer cell wall of Salmonella typhimurium in ensuring resistance to the drug. Int $J$ Antimicrob Agents. 2000;14 (3):225-229.

19. Amaral L, Kristiansen JE, Fau - Abebe LS, Abebe LS, Fau Millett W, Millett W. Inhibition of the respiration of multi-drug resistant clinical isolates of Mycobacterium tuberculosis by thioridazine: potential use for initial therapy of freshly diagnosed tuberculosis. J Antimicrob Chemother. 1996;38(6):1049-1053.
20. Cao Y, Naseri M, He Y, Xu C, Walsh LJ, Ziora ZM. Non-antibiotic antimicrobial agents to combat biofilm-forming bacteria. $J$ Glob Antimicrob Resist. 2020;21:445-451. doi:10.1016/j.jgar.2019.11.012

21. Opal SM. Non-antibiotic treatments for bacterial diseases in an era of progressive antibiotic resistance. Crit Care. 2016;20(1):397. doi:10.1186/s13054-016-1549-1

22. Dastidar SG, Fau - Saha PK, Saha PK, et al. Antibacterial activity of ambodryl and benadryl. J Appl Microbiol. 1976;41(2):209-214.

23. Molnár J, Fau - Mándi Y, Mándi Y, Fau - Király J, Király J. Antibacterial effect of some phenothiazine compounds and R-factor elimination by chlorpromazine. Acta Microbiol Acad Sci Hung. 1976;23(1):45-54.

24. Nasrin F. Study of antimicrobial and antioxidant potentiality of anti-diabetic drug metformin. Int J Pharm Drug Anal. 2014:220-224.

25. Duncan AI, Koch CG, Xu M, et al. Recent metformin ingestion does not increase in-hospital morbidity or mortality after cardiac surgery. Anesth Analg. 2007;104(1):42-50. doi:10.1213/01.ane.00002425 32.42656.e7

26. Shih C-J, Wu Y-L, Chao P-W, et al. Association between use of oral anti-diabetic drugs and the risk of sepsis: a Nested Case-Control Study. Sci Rep. 2015;5(1):15260. doi:10.1038/srep15260

27. Mor A, Petersen I, Sørensen HT, Thomsen RW. Metformin and other glucose-lowering drug initiation and rates of community-based antibiotic use and hospital-treated infections in patients with type 2 diabetes: a Danish nationwide population-based cohort study. $B M J$ Open. 2016;6(8):e011523. doi:10.1136/bmjopen-2016-011523

28. Liu Y, Jia Y, Yang K, et al. Metformin restores tetracyclines susceptibility against multidrug resistant bacteria. Adv Sci. 2020;7 (12):1902227. doi:10.1002/advs.201902227

29. Xiao Y, Liu F, Li S, et al. Metformin promotes innate immunity through a conserved PMK-1/p38 MAPK pathway. Virulence. 2020;11(1):39-48. doi:10.1080/21505594.2019.1706305

30. Komatsuzawa H, Ohta K, Fau - Sugai M, et al. Tn551-mediated insertional inactivation of the fmtB gene encoding a cell wall-associated protein abolishes methicillin resistance in Staphylococcus aureus. J Antimicrob Chemother. 2000;45(4):421-431.

31. Andrews JM. Determination of minimum inhibitory concentrations. J Antimicrobial Chemother. 2001;48(suppl_1):5-16.

32. Al Tall Y, Abualhaijaa A, Alsaggar M, Almaaytah A, Masadeh M, Alzoubi KH. Design and characterization of a new hybrid peptide from LL-37 and BMAP-27. Infect Drug Resist. 2019;12:1035-1045. doi:10.2147/IDR.S199473

33. Ammerman NC, Beier-Sexton M, Azad AF. Growth and maintenance of Vero cell lines. Curr Protoc Microbiol. 2008;Appendix 4: Appendix-4E. doi:10.1002/9780471729259.mca04es11

34. Singhal A, Jie L, Kumar P, et al. Metformin as adjunct antituberculosis therapy. Sci Transl Med. 2014;6(263):263ra159. doi:10.1126/ scitranslmed.3009885

35. Gupta PD, Birdi TJ. Development of botanicals to combat antibiotic resistance. J Ayurveda Integr Med. 2017;8(4):266-275. doi:10.1016/j. jaim.2017.05.004

36. Boucher HW, Talbot GH, Bradley JS, et al. Bad bugs, no drugs: no ESKAPE! An update from the Infectious Diseases Society of America. Clin Infect Dis. 2009;48(1):1-12. doi:10.1086/595011

37. Baquero F, Coque TM, de la Cruz F. Ecology and evolution as targets: the need for novel eco-evo drugs and strategies to fight antibiotic resistance. Antimicrob Agents Chemother. 2011;55 (8):3649. doi:10.1128/AAC.00013-11

38. Thanacoody HK. Thioridazine: resurrection as an antimicrobial agent? Br J Clin Pharmacol. 2007;64(5):566-574.

39. Melander RJ, Melander C. The challenge of overcoming antibiotic resistance: an adjuvant approach? ACS Infect Dis. 2017;3 (8):559-563. doi:10.1021/acsinfecdis.7b00071

40. Kristiansen JE, Mortensen I. Antibacterial effect of four phenothiazines. Pharmacol Toxicol. 1987;60(2):100-103. doi:10.11 11/j.1600-0773.1987.tb01504.x 
41. Ejim L, Farha MA, Falconer SB, et al. Combinations of antibiotics and nonantibiotic drugs enhance antimicrobial efficacy. Nat Chem Biol. 2011;7(6):348-350. doi:10.1038/nchembio.559

42. Pernicova I, Korbonits M. Metformin-mode of action and clinical implications for diabetes and cancer. Nat Rev Endocrinol. 2014;10 (3):143-156. doi:10.1038/nrendo.2013.256

43. Siavash M, Tabbakhian M, Sabzghabaee AM, Razavi N. Severity of gastrointestinal side effects of metformin tablet compared to metformin capsule in type 2 diabetes mellitus patients. J Res Pharm Pract. 2017;6(2):73-76. doi:10.4103/jrpp.JRPP_17_2

44. American Diabetes Association. Type $\overline{2}$ diabetes in children and adolescents. Pediatrics. 2000;105(3):671-680. doi:10.1542/ peds.105.3.671

45. Scarpello JH, Howlett HC. Metformin therapy and clinical uses. Diabetes Vasc Dis Res. 2008;5(3):157-167. doi:10.3132/ dvdr.2008.027

46. Vashisht R, Brahmachari SK. Metformin as a potential combination therapy with existing front-line antibiotics for tuberculosis. $J$ Transl Med. 2015;13(1):83. doi:10.1186/s12967-015-0443-y

47. Gill SK, Hui K, Farne H, et al. Increased airway glucose increases airway bacterial load in hyperglycaemia. Sci Rep. 2016;6(1):1.

48. Dash AK, Behera SR, Pattanaik BK, Palo AK. Study of antimicrobial property of some hypoglycemic drugs. Chron Young Sci. 2011;2 (4):219. doi:10.4103/2229-5186.93029

49. Marupuru S, Senapati P, Pathadka S, Miraj SS, Unnikrishnan MK, Manu MK. Protective effect of metformin against tuberculosis infections in diabetic patients: an observational study of south Indian tertiary healthcare facility. Braz J Infect Dis. 2017;21(3):312-316.

50. Singhal A, Jie L, Kumar P, et al. Metformin as adjunct antituberculosis therapy. Sci Transl Med. 2014;6(263):263ra159.

51. Nasri H, Rafieian-Kopaei M. Metformin: current knowledge. J Res Med Sci. 2014;19(7):658-664.
52. Hundal RS, Inzucchi SE. Metformin: new understandings, new uses. Drugs. 2003;63(18):1879-1894.

53. Scarpello JH, Howlett HC. Metformin therapy and clinical uses. Diabetes Vasc Dis Res. 2008;5(3):157-167.

54. Śmieszek A, Basińska K, Chrząstek K, Marycz K. In vitro and in vivo effects of metformin on osteopontin expression in mice adipose-derived multipotent stromal cells and adipose tissue. $J$ Diabetes Res. 2015;2015:814896. doi:10.1155/2015/814896

55. Ullah A, Ashraf M, Javeed A, Anjum AA, Attiq A, Ali S. Enhancement of anti-proliferative activities of metformin, when combined with celecoxib, without increasing DNA damage. Environ Toxicol Pharmacol. 2016;45:227-234.

56. Foretz M, Guigas B, Bertrand L, Pollak M, Viollet B. Metformin: from mechanisms of action to therapies. Cell Metab. 2014;20 (6):953-966. doi:10.1016/j.cmet.2014.09.018

57. Sant'Anna JR, Yajima JP, Fau - Rosada LJ, et al. Metformin's performance in in vitro and in vivo genetic toxicology studies. Exp Biol Med. 2013;238(7):803-810.

58. Al-Janabi AAHS. In vitro antibacterial activity of Ibuprofen and acetaminophen. J Glob Infect Dis. 2010;2(2):105-108. doi:10.4103/ 0974-777X.62880

59. Poulsen M, Klitgaard JK, Christensen JB, et al. Comparison of antibacterial activity of (-) thioridazine and racemic thioridazine in Staphylococcus aureus. Am J Bioequivalence Bioavailab. 2018;1 (1):1-9.

60. Nikaido H. Molecular basis of bacterial outer membrane permeability revisited. Microbiol Mol Biol Rev. 2003;67(4):593-656.

61. Kajiwara C, Kusaka YA-O, Kimura S, et al. Metformin mediates protection against legionella pneumonia through activation of AMPK and mitochondrial reactive oxygen species. J Immunol. 2018;200(2):623-631.

\section{Publish your work in this journal}

Clinical Pharmacology: Advances and Applications is an international, peer-reviewed, open access journal publishing original research, reports, reviews and commentaries on all areas of drug experience in humans. The manuscript management system is completely online and includes a very quick and fair peer-review system, which is all easy to use. Visit http://www.dovepress.com/testimonials.php to read real quotes from published authors. 